\title{
Unraveling AGN feedback and ICM physics with deep Chandra X-ray observations of the galaxy group NGC 5813
}

\author{
Scott W. Randall ${ }^{1}$, Paul E. J. Nulsen ${ }^{1}$, Christine Jones ${ }^{1}$, \\ William R. Forman ${ }^{1}$, Tracy E. Clarke ${ }^{2}$ and Elizabeth L. Blanton ${ }^{3}$ \\ ${ }^{1}$ Harvard-Smithsonian Center for Astrophysics, \\ 60 Garden St., Cambridge, MA, 02138, USA \\ email: srandall@cfa.harvard.edu, pnulsen@cfa.harvard.edu, \\ cjones@cfa.harvard.edu, wforman@cfa.harvard.edu \\ ${ }^{2}$ Naval Research Laboratory, \\ Code 7213, 4555 Overlook Ave SW, Washington, DC 20375, USA \\ email: tracy.clarke@nrl.navy.mil \\ ${ }^{3}$ Astronomy Department and Institute for Astrophysical Research, Boston University, \\ 725 Commonwealth Avenue, Boston, MA 02215, USA \\ email: eblanton@bu.edu
}

\begin{abstract}
We present results from deep Chandra X-ray observations of the galaxy group NGC 5813. This system shows three pairs of collinear cavities, with each pair associated with an elliptical AGN outburst shock. Due to the relatively regular morphology of this system, and the unique unambiguous detection of three distinct AGN outburst shocks, it is particularly wellsuited for the study of AGN feedback and the AGN outburst history. We find that the mean kinetic power is roughly the same for each outburst, and that the total energy associated with the youngest outburst is significantly lower than that of the previous outbursts. This implies that the mean AGN jet power has remained stable for at least $50 \mathrm{Myr}$, and that the youngest outburst is ongoing. We find that the mean shock heating rate balances the local radiative cooling rate at each shock front, suggesting that AGN outburst shock heating alone is sufficient to offset cooling and establish AGN/ICM feedback within at least the central $30 \mathrm{kpc}$. Finally, we find non-zero shock front widths that are too large to be explained by particle diffusion, but are instead consistent with arising from broadening of the shock fronts due to propagation through a turbulent ICM with a mean turbulent speed of $\sim 70 \mathrm{~km} \mathrm{~s}^{-1}$.
\end{abstract}

Keywords. X-rays: individual (NGC 5813), X-rays: galaxies, X-rays: galaxies: clusters

\section{Introduction}

Early Chandra and XMM-Newton X-ray observations revealed that the amount of gas cooling to very low temperatures at the centers of cool core clusters is much less than what is expected from simple radiative cooling models (David et al. 2001; Peterson et al. 2001; Peterson \& Fabian 2006). The implication is that the diffuse X-ray emitting gas must be heated, either by pre-heating during cluster formation or by ongoing energy injection. The most likely heating mechanism is feedback due to energy injection by the central AGN of the cD galaxy (see McNamara \& Nulsen 2007 and Fabian 2012 for recent reviews). During this process, matter is accreted onto the central supermassive black hole (SMBH), which drives powerful jets. These jets evacuate cavities in the intracluster medium (ICM), which can drive shocks as they are inflated and subsequently rise buoyantly (Churazov et al. 2001). The energy contained in cavities and shocks is then free to heat the ICM, which lowers the cooling rate and subsequently the $\mathrm{SMBH}$ accretion rate. The ensuing 


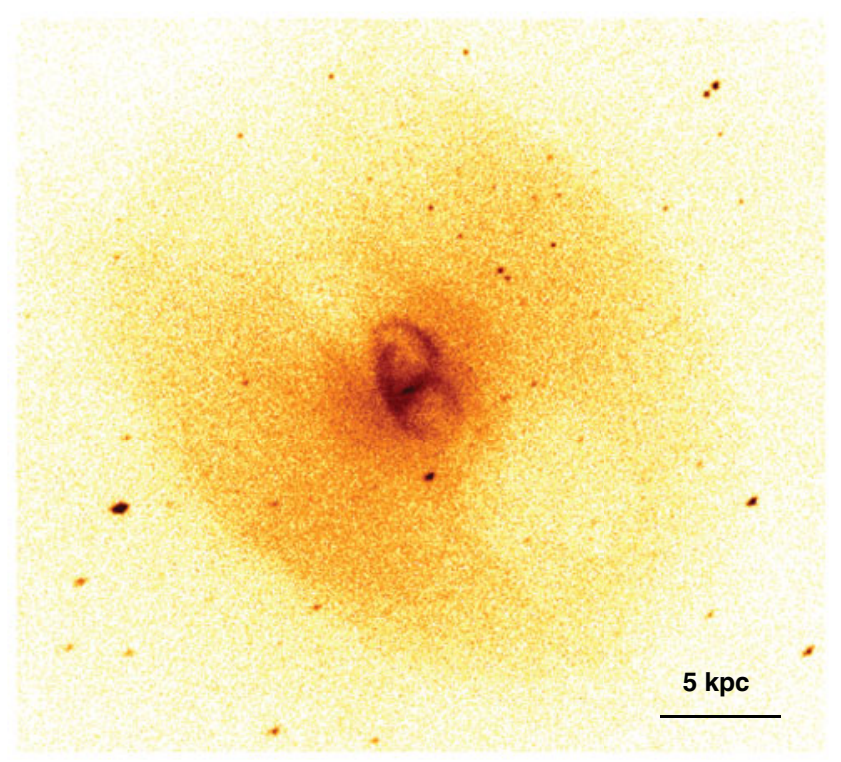

Figure 1. Exposure corrected, background subtracted, $0.3-3 \mathrm{keV}$ Chandra image of the central region of $\mathrm{N} 5813$, unsmoothed and with point sources included (1 pixel $=0.5 ")$.

decrease in AGN heating allows the gas to once again cool and accrete onto the SMBH, establishing a feedback loop that regulates the temperature of the ICM. Several studies have shown that, generally, the total enthalpy in cavities in cool core systems is sufficient to offset radiative cooling in individual galaxies, galaxy groups, and clusters (Bîrzan et al. 2004; Rafferty et al. 2006; Nulsen et al. 2007; Hlavacek-Larrondo et al. 2012). However, the details of how and where this energy gets transferred to the ICM are unclear. Weak AGN outburst shocks are also expected to heat the ICM, although they are difficult to detect and unambiguous examples are very rare.

Here we report on results from a very deep Chandra observation of the central galaxy in the relatively isolated galaxy group NGC 5813 (N5813). The ICM in this group has a remarkably regular morphology, with three pairs of roughly collinear cavities, and each pair associated with an elliptical shock front edge. With clear, cleanly separated signatures from three distinct outbursts of the central AGN and no other significant dynamical processes at work, N5813 is uniquely well-suited to the study of AGN feedback. In this work, we focus on the implications for AGN feedback, the outburst history of the central SMBH, and ICM transport processes. In Randall et al. (2011) we presented results based on an initial $150 \mathrm{ks}$ Chandra observation of N5813. In this proceedings article, we summarize some results from recent, deeper Chandra observations with a total of $650 \mathrm{ks}$ of observing time. Further results and details are given in Randall et al. (2014, hereafter R14).

\section{X-ray observations}

Details on the data analysis and creation of the X-ray maps are given in R14. Fig. 1 shows the unsmoothed Chandra image of the core of N5813. Bright rims surround an inner pair of cavities at $\sim 1 \mathrm{kpc}$. An elliptical shock at $\sim 10 \mathrm{kpc}$ surrounds, but is detached from, a second pair of cavities. The smoothed image with point sources removed and residual image (Fig. 2) reveal a third pair of cavities, associated with a third shock front 

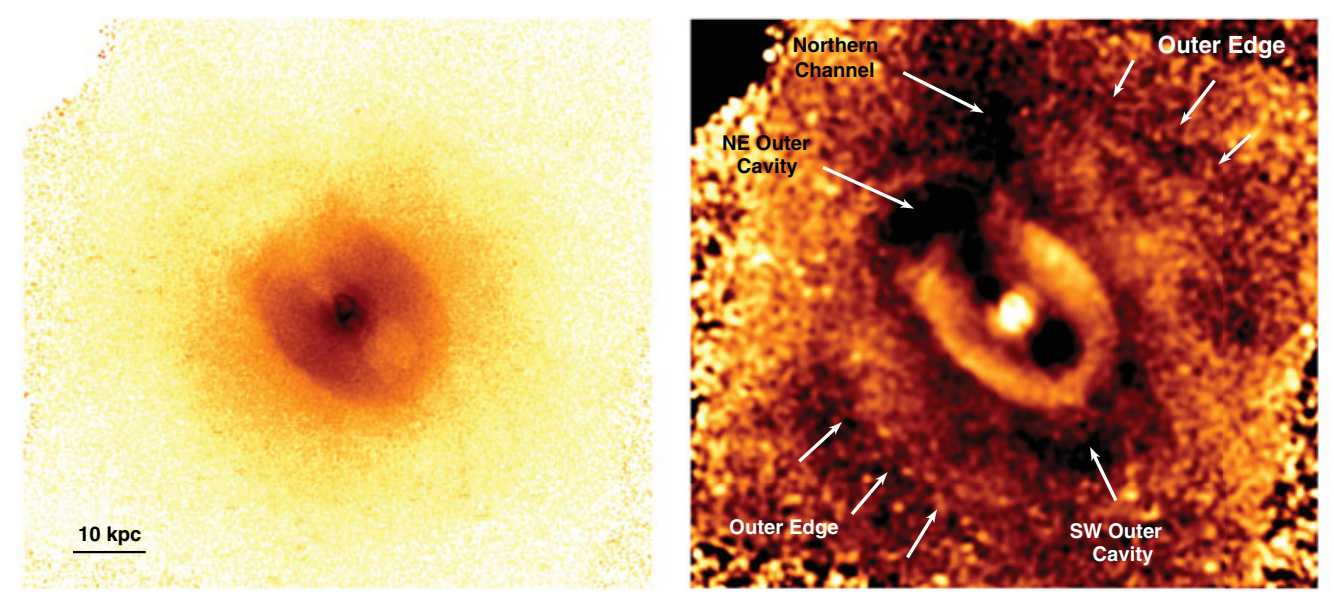

Figure 2. Left: Exposure corrected, background subtracted, $0.3-3 \mathrm{keV}$ Chandra image, with point sources removed and smoothed with a $\sigma=1.5 "$ Gaussian. The image shows bright rims surrounding an inner pair of cavities, a prominent elliptical edge surrounding a pair of cavities at intermediate radii (with the more obvious cavity to the SW and the NE cavity apparently broken up into two connected cavities), and a subtle outer edge associated with a faint pair of outer cavities (with the more obvious cavity to the NE). Right: X-ray image divided by a $2 \mathrm{D}$ fitted beta model and smoothed with a $\sigma=6$ " Gaussian, shown on the same scale. The outer cavities and edges are more clearly seen in this residual image, while the inner cavities are not visible due to the larger smoothing scale and saturation of the color scale. The image also reveals a faint "channel" of decreased surface brightness extending to the north, apparently connected to the NE outer cavity.

at $\sim 30 \mathrm{kpc}$. The temperature maps (Fig. 3) show clear temperature increases at the $10 \mathrm{kpc}$ and $1 \mathrm{kpc}$ shock fronts, and hint at increases at the $30 \mathrm{kpc}$ fronts, confirming these features as shocks (as opposed to cold fronts). Detailed temperature, pressure, and density profiles across each edge further confirm them as shock fronts, with Mach numbers of roughly $1.8,1.5$, and 1.3 for the $1 \mathrm{kpc}, 10 \mathrm{kpc}$, and $30 \mathrm{kpc}$ shocks, respectively (R14).

\section{Shock heating and feedback}

Shocks are expected to heat the ICM as they propagate, offsetting radiative cooling. The relevant quantity for heating is not the transient rise in temperature at the shock front, but the change in entropy $\Delta S$. The equivalent amount of heat energy imparted to the gas by a shock due to an entropy change of $\Delta S$ is given by

$$
\Delta Q \simeq T \Delta S=E \Delta \ln \frac{p}{\rho^{\gamma}},
$$

where $E=C_{\mathrm{V}} T$ is the total thermal energy of the gas, $\gamma$ is the adiabatic index (taken to be 5/3), and $p$ and $\rho$ are the local pressure and density, respectively (Nulsen et al. 2007; Randall et al. 2011; R14). Thus, each shock contributes a fraction $\Delta \ln \frac{p}{\rho^{\gamma}}$ of the total thermal energy in the gas. In the case of weak shocks, this fraction is small. To consider the cumulative effect of repeated weak shocks, we compare the outburst repetition rate to the local radiative cooling rate at each shock front, where the former is taken to be one outburst every $20 \mathrm{Myr}$ based on the shock locations and Mach numbers, and the latter is estimated from the observed X-ray luminosity. 

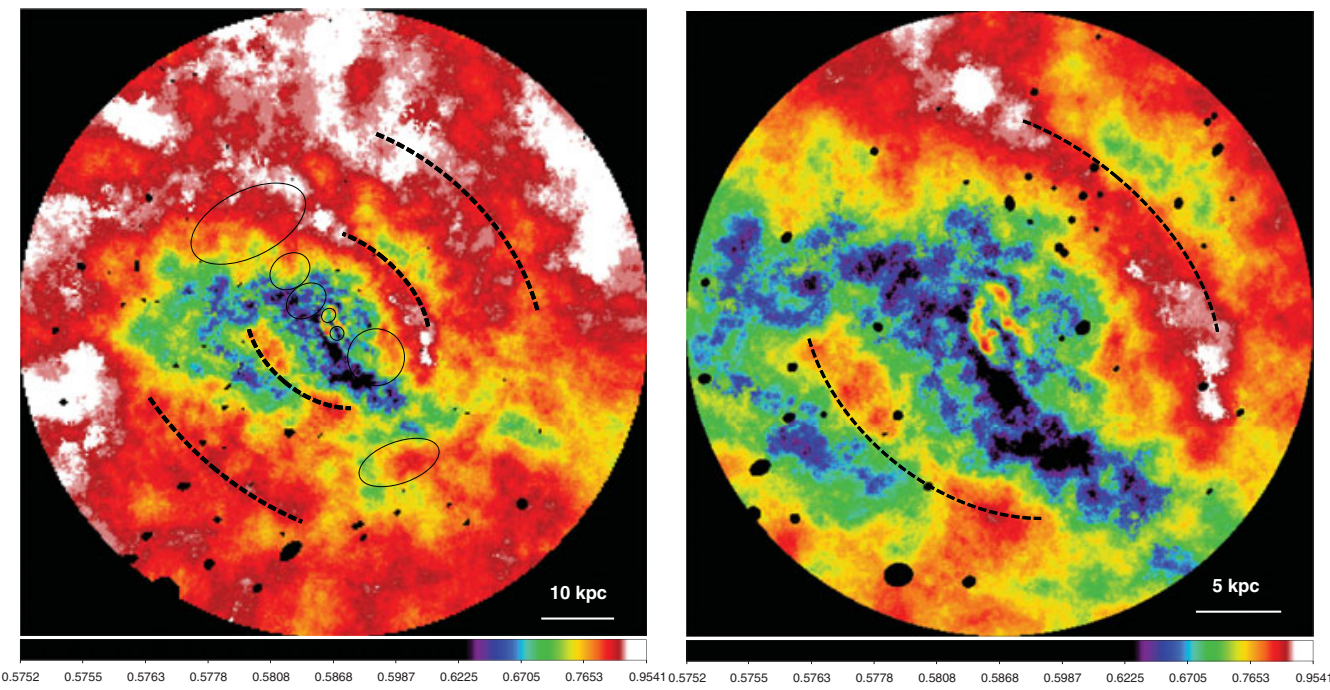

Figure 3. Left: Smoothed temperature map. The locations of the $10 \mathrm{kpc}$ and $30 \mathrm{kpc}$ shock fronts are indicated with dashed black lines, and the cavity locations with black ellipses. The map clearly shows temperature increases associated with the $1 \mathrm{kpc}$ and $10 \mathrm{kpc}$ shocks, and hints at increases associated with the $30 \mathrm{kpc}$ shock (particularly to the NW). Also visible is a SW to NE plume of cool gas that has been uplifted by the buoyantly rising cavities. Right: High-resolution temperature map of the core region, with the $10 \mathrm{kpc}$ shock fronts indicated as on the left.

We find that roughly 9, 21, and 143 shocks are required per local cooling time to completely offset radiative cooling in the gas, whereas 7, 46, and 111 are expected for an outburst repetition rate of one every $20 \mathrm{Myr}$ for the $1 \mathrm{kpc}, 10 \mathrm{kpc}$, and $30 \mathrm{kpc}$ shocks, respectively. Despite being rough estimates, in each case the number of required shocks agrees with the number expected remarkably well. We conclude that shock heating alone is sufficient to balance radiative cooling in the gas out to at least $\sim 30 \mathrm{kpc}$. This heating is more effective at smaller radii and takes place roughly isotropically, both of which are required for AGN feedback to operate. The cavities will continue to rise buoyantly and deposit their internal energy into the ICM at larger radii. We suggest that AGN outburst shocks may generally play a significant role in AGN feedback, particularly at small radii where the Mach numbers are higher. These weak shocks are generally difficult to detect, since the shock fronts are thin and easily masked by projection effects, particularly in systems with complicated structure in the ICM. They are more easily detected in N5813 due to its proximity, low temperature, and regular morphology.

\section{AGN outburst history}

The power output of the central AGN is dominated by its kinetic luminosity. Since the kinetic energy output by the AGN drives cavities and shocks in the ICM we can use X-ray observations of these features to constrain the outburst history of the AGN. We take a cavity's internal energy to be $3 p V$, where $p$ is the average pressure at the cavity radius and $V$ is the cavity volume. The shock energy is estimated as $E_{\mathrm{s}} \approx p_{\mathrm{s}} V_{\mathrm{s}}\left(p_{2} / p_{1}-1\right)$, where $p_{1}$ and $p_{2}$ are the pre- and post-shock pressures, respectively, $V_{\mathrm{s}}$ is the total volume enclosed by the shock front, and $p_{\mathrm{s}}$ is the average pressure within $V_{\mathrm{s}}(\mathrm{R} 14)$. 
Using the above estimates, we find roughly equal total outburst energies for the outbursts associated with the $10 \mathrm{kpc}$ and $30 \mathrm{kpc}$ shocks, and a total energy that is more than an order of magnitude less for the outburst associated with the $1 \mathrm{kpc}$ shock. If we divide the total energy of each outburst by its duration, we find that the average power is roughly the same for each outburst, at $2 \times 10^{43} \mathrm{erg} \mathrm{s}^{-1}$. This implies that the current outburst is ongoing, actively inflating cavities that are driving shocks into the ICM, and that the mean outburst power (and hence, the mean kinetic jet power) can remain stable over long timescales (in this case, for at least $50 \mathrm{Myr}$ ).

\section{ICM transport processes}

When fitting density profiles to the projected shock front edges, we find that a model with a Gaussian smoothed (as opposed to discontinuous) edge is statistically preferred for the $10 \mathrm{kpc}$ and $1 \mathrm{kpc}$ shock fronts (for the $30 \mathrm{kpc}$ shock fronts only upper limits on the smoothing scale can be placed). This broadening is too large to be explained by blurring due to the PSF (R14). The fitted shock front widths can be compared with the particle mean free path lengths $(\lambda)$ across the shock fronts. Broadening due to particle diffusion should lead to shock fronts that are 1.5-2 times $\lambda$ (Landau \& Lifshitz 1987). We find widths that are an order of magnitude or more larger than the local values of $\lambda$, at better than $90 \%$ confidence, suggesting that the shocks are broadened by something other than particle diffusion (e.g., projection effects, systematic effects due to uncertain shock front geometries, propagation through a clumpy and/or turbulent ICM).

Nulsen et al. (2013) provide an estimate of the expected shock width due to turbulence as a function of radius as a shock propagates through a uniformly turbulent ICM. We invert this relation to give the rms turbulent speed implied by the observed broadening,

$$
\sigma_{\mathrm{t}} \approx \frac{w v_{\mathrm{s}}}{\sqrt{r_{\mathrm{s}} \ell}}
$$

where $w$ is the shock width, $r_{\mathrm{s}}$ is the shock radius, and $\ell$ is the turbulence coherence length (which we take to be $0.1 r_{\mathrm{s}}$, see Nulsen et al. 2013, Rebusco et al. 2005). We find that each shock front width is consistent with a turbulent speed of $\sigma_{\mathrm{t}} \approx 70 \mathrm{~km} \mathrm{~s}{ }^{-1}$, which is reasonable compared with results from simulations (e.g., Lau et al. 2009) and independent methods using other observations (e.g. , de Plaa et al. 2012; Sanders \& Fabian 2013). While we can not rule out broadening due to projection and other effects (mentioned above), these effects will only increase the apparent shock front widths. Thus, our results on the turbulent speed represents an upper limit, which is already on the low end of the typical range of $100-300 \mathrm{~km} \mathrm{~s}^{-1}$ expected based on other studies.

\section{Acknowledgements}

Support for this work was partially provided by the Chandra X-ray Center through NASA contract NAS8-03060, the Smithsonian Institution, and by Chandra X-ray observatory grant GO1-12104X.

\section{References}

Bîrzan, L., Rafferty, D. A., McNamara, B. R., Wise, M. W., \& Nulsen, P. E. J. 2004, ApJ, 607, 800

Churazov, E., Brüggen, M., Kaiser, C. R., Böhringer, H., \& Forman, W. 2001, ApJ, 554, 261

David, L. P., Jones, C., Forman, W., et al. 2009, ApJ, 705, 624

de Plaa, J., Werner, N., Simionescu, A., et al. 2010, A\& $A, 523,81$

Fabian, A. C. 2012, ARAEAA, 50, 455 
Hlavacek-Larrondo, J., Fabian, A. C., Edge, A. C., et al. 2012, MNRAS, 421, 1360

Landau L. D., \& Lifshitz E. M. 1987 Course of Theoretical Physics, Vol. 6: Fluid Mechanics (London: Pergamon)

Lau, E. T., Kravtsov, A. V., \& Nagai, D. 2009, ApJ, 705, 1129

McNamara, B. R. \& Nulsen, P. E. J. 2007, ARA\&A, 45, 117

Nulsen, P. E. J., Jones, C., Forman, W. R., et al. 2007, in Heating versus Cooling in Galaxies and Clusters of Galaxies, ed. H. Böhringer, G. W. Pratt, A. Finoguenov, \& P. Schuecker (Berlin: Springer), 210

Nulsen, P. E. J., Li, Z., Forman, W. R., et al. 2013, ApJ, 775, 117

Peterson, J. R. \& Fabian, A. C. 2006, PhR, 427, 1

Peterson, J. R., Paerels, F. B. S., Kaastra, J. S., et al. 2001, A $\&$ A, 365, L104

Rafferty, D. A., McNamara, B. R., Nulsen, P. E., \& Wise, M. W. 2006, ApJ, 652, 216

Randall, S. W., Forman, W. R., Giacintucci, S., et al. 2011, ApJ, 726, 86

Randall, S. W. et al. 2014, in preparation

Rebusco, P., Churazov, E., Böhringer, H., \& Forman, W. 2005, MNRAS, 359, 1041

Sanders, J. S. \& Fabian, A. C. 2013, MNRAS, 429, 2727 